\title{
Effect of Vibrations with Different Frequencies on Reduction of Residual Stress of Welded Joint**
}

\author{
Shugeru AOKI**, Tadashi NISHIMURA**, Tetsumaro HIROI** \\ and Seiji HIRAI*** \\ **Tokyo Metropolitan College of Industrial Technology \\ 1-10-40 Higashi-Ohi, Shinagawa-ku, Tokyo, Japan \\ E-mail:aoki@Tokyo-tmct.ac.jp \\ *** Institute of Technologists \\ 333 Maeya, Gyoda-city, Saitama, Japan
}

\begin{abstract}
Welding is widely used for construction of many structures. It is well known that residual stress is generated near the bead because base metal is heated near the bead. Tensile residual stress on the surface degrades fatigue strength. Some reduction methods of residual stress are practically used, for example, heat treatment and shot peening. The authors developed a new method for reduction of residual stress using vibration during welding. In this method, single vibration was used. The effectiveness of the method was demonstrated. In this paper, the effect of vibrations with different frequencies on reduction of residual stress is examined. The effect is examined experimentally by butt-welding of thin plates. First, two thin plates are butt-welded using ultrasonic vibrations with different frequencies on each plate. Some plates are welded using single ultrasonic vibration and without ultrasonic vibration for comparison. When thin plates are welded using vibrations with different frequencies, tensile residual stresses are reduced and reduction rate is largest compared with other conditions. Second, two thin plates are butt-welded using ultrasonic vibration and vibration with low frequency. Some plates are welded using single vibration and without vibration for comparison. In this case, tensile residual stresses are reduced and reduction rate is largest compared with other conditions. Obtained results are examined by analytical method.
\end{abstract}

Key words: Welding, Ultrasonic Vibration, Residual Stress, Frequency, X-Ray Diffraction Method, Thin Plate, Elasto-Plastic Model

\section{Introduction}

Welding is widely used for construction of many structures. It is well known that residual stress is generated near the bead ${ }^{(1)}$ since welding is joining method using locally given heat. Tensile residual stress on the surface of metal degrades fatigue strength. Some reduction methods of residual stress are examined ${ }^{(2)}$. As practical methods, heat treatment and shot peening are used $^{(3)}$. In those methods, there are some problems, that is, requirement of special equipment, time consuming and degradation of surface of metal. Reduction method using vibration after welding was proposed. However, the effectiveness of this method is not clear ${ }^{(4)}$.

The authors proposed a new reduction method using vibration during welding and examined the effectiveness of the proposed method. They showed that tensile residual stress is reduced near the bead using vibration with relatively low frequency near the natural frequency of the material ${ }^{(5),(6)}$. They also showed that tensile residual stress is reduced using random vibration having wide range of frequencies ${ }^{(7)}$.

On the other hand, ultrasonic vibration is transmitted in the solid effectively. Ultrasonic vibration is widely used in manufacturing, for example, cutting hard metal and plastic formation $^{(8)}$. Considering above mentioned points, the authors proposed a method using

${ }^{*}$ Received 31 Oct., 2007 (No. 07-0669) [DOI: 10.1299/jmmp.2.428] 
ultrasonic vibration during welding and showed that tensile residual stress is reduced ${ }^{(9)}$. In this method, two thin plates are butt-welded and ultrasonic vibration is applied to one plate (9). It is expected that the effect of reduction of residual stress is larger when ultrasonic vibrations apply to both plates. However, when both frequencies are equal, ultrasonic vibrators may be destroyed because of resonant phenomenon.

In this paper, the effect of vibrations with different frequencies on butt-welding of two thin plates is examined. First, one ultrasonic vibration is applied on one plate and the other ultrasonic vibration with different frequency is applied on the other thin plate. Second, one ultrasonic vibration is applied on one plate and the other vibration with relatively low frequency which is the fundamental natural frequency of the thin plate is applied on both plates. Finally, experimental results are examined analytically. Residual stress is obtained from simulation method using an analytical model considering metal near the bead.

\section{Experiment Using Ultrasonic Vibration with Different Frequencies}

When two thin plates are butt-welded, an ultrasonic vibration is applied on one plate and the other ultrasonic vibration with different frequency is applied on the other plate. Reduction of residual stress is examined in this case.

\subsection{Experimental setup}

Figure 1 shows size of specimen. Material of specimen is the rolled steel for general structure (JIS SS400). In order to eliminate residual stress induced by rolling, it was annealed at $900^{\circ} \mathrm{C}$ for one hour and cooled in a furnace until it was $200^{\circ} \mathrm{C}$. The groove is $\mathrm{V}$-shaped and the groove angle is $45^{\circ}$. Root opening is $1.7 \mathrm{~mm}$. Two thin plates shown in Fig. 1 are hold onto the supporting device as shown in Fig.2 and shaken by ultrasonic vibration during welding. Specimens are welded using an automatic acid gas shielded arc welding machine. Welding is completed through one pass. As shown in Fig.2, ultrasonic vibrator is put on the center line and $20 \mathrm{~mm}$ from edge of each thin plate. If frequencies of both ultrasonic vibrators are same, one ultrasonic vibrator is destroyed because of resonance. Then frequencies of ultrasonic vibration are chosen as $60 \mathrm{kHz}$ and $27.5 \mathrm{kHz}$. Output power of each ultrasonic vibrator is $100 \mathrm{~W}$. The natural frequency of specimen hold onto the supporting device as shown in Fig. 2 before welding is $35 \mathrm{~Hz}$. For comparison, some specimens are welded using one ultrasonic vibration on one plate. Some specimens are welded without vibration.

Welding speed is $3.75 \mathrm{~mm} / \mathrm{s}$. Welding is completed in 27 second. Diameter of wire is $0.9 \mathrm{~mm}$. Voltage is $21 \mathrm{~V}$ and current is $200 \mathrm{~A}$.

Residual stress is measured using X-ray diffractometer with a scintillation counter after removing quenched scale chemically using hydrochloric acid having a concentration of $6 \mathrm{~mol} / \ell$ and smoothing surface of the bead using CPL (chemical polishing liquid). Table 1 shows measuring conditions of residual stress.

Figure 3 shows measuring locations of residual stress. Residual stresses are measured at 9 points on the bead (y-axis) and at 11 points on the center line perpendicular to the bead (x-axis). Residual stresses in direction of the bead are measured.

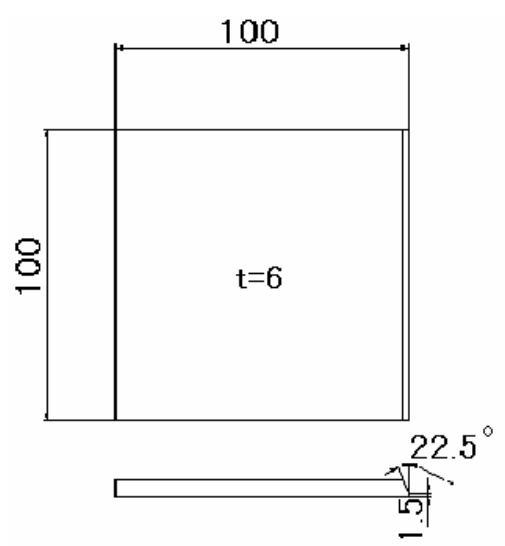

Fig.1 Size and shape of specimen (mm) 

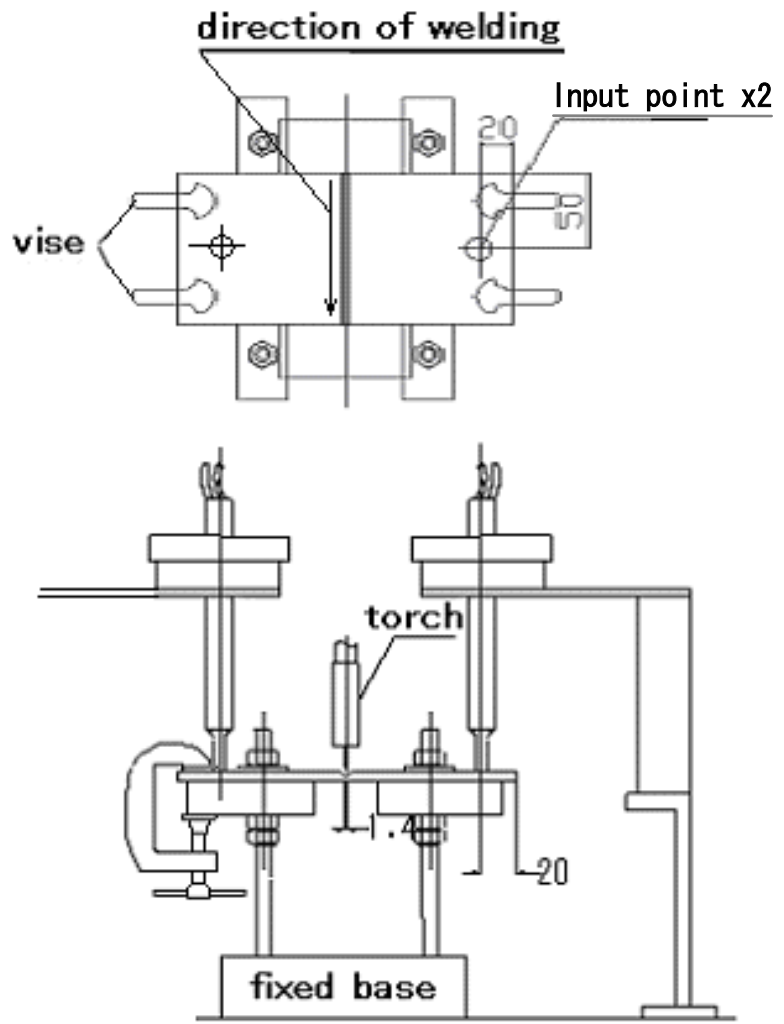

Fig.2 Set of specimens (mm)

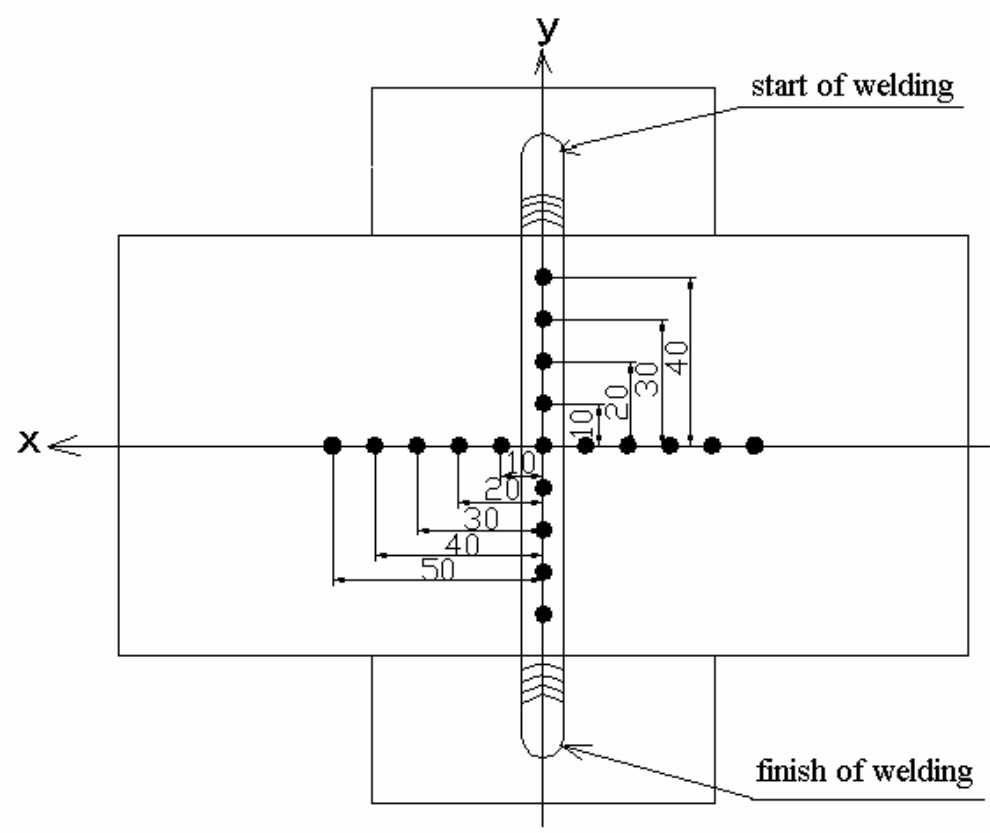

Fig.3 Measuring locations of residual stress (mm) 


\subsection{Results of Experiment}

In Fig.4 and Fig.5, residual stresses on the bead (y-axis) and on the center line perpendicular to the bead (x-axis), respectively. $\bigcirc$ shows residual stresses of specimen welded without ultrasonic vibration.

shows residual stress using $60 \mathrm{kHz}$ ultrasonic vibration on one plate. shows residual stress using $27.5 \mathrm{kHz}$ ultrasonic vibration on one plate.

shows residual stress using $60 \mathrm{kHz}$ ultrasonic vibration on one plate and $27.5 \mathrm{kHz}$ ultrasonic vibration on the other one plate.

Figure 4 shows residual stresses on the bead. Tensile residual stresses are measured at almost of all points. Especially, high tensile residual stress is measured at center of the bead. When ultrasonic vibration is applied on one plate, tensile residual stress is significantly reduced at center of the bead. In this case, the effect of frequency on reduction of residual stress is not different for $60 \mathrm{kHz}$ and $27.5 \mathrm{kHz}$. The same phenomenon is observed when frequency of vibration is relatively low ${ }^{(6)}$. On the other hand, when ultrasonic vibrations are applied on both plates, reduction rate is larger than one excitation.

Figure 5 shows residual stress on the line perpendicular to the bead. Tensile residual stresses are measured $10 \mathrm{~mm}$ from the bead. When ultrasonic vibration is used, residual stress is reduced in this region. Especially, when ultrasonic vibrations with different frequencies are used on both plates, reduction rate of residual stresses is large.

From both figures, when ultrasonic vibration is applied on one plate, tensile residual stress is reduced at center of the bead. In this case, the effect of frequency is not observed. On the other hand, when ultrasonic vibrations are applied on both plates, reduction rate of residual stresses is large.

3. Experiment Using Ultrasonic Vibration and Low Frequency Vibration

When thin plates are butt-welded, ultrasonic vibration is applied on one plate and low
Table 1 Conditions of $\mathrm{X}$ ray stress measurements

\begin{tabular}{|l|l|}
\hline Characteristic X-rays & $\mathrm{Cr}-\mathrm{K} \alpha$ \\
\hline Diffraction plane & $\alpha-\mathrm{Fe}(211)$ \\
\hline Filter & Vanadium foil \\
\hline Stress determination & $\sin ^{2} \psi$ method \\
\hline Psi angle & $0^{\circ}, 15^{\circ}, 30^{\circ}, 45^{\circ}$ \\
\hline Irradiated area & $2 \mathrm{x} 4 \mathrm{~mm}^{2}$ \\
\hline $\begin{array}{l}\text { Tube voltage and } \\
\text { current }\end{array}$ & $30 \mathrm{kV}, 8 \mathrm{~mA}$ \\
\hline Scan condition & $1.0^{\circ}$ \\
\hline Peak determination & $\begin{array}{l}\text { Half value width } \\
\text { method }\end{array}$ \\
\hline $\begin{array}{l}\text { Direction } \\
\text { measurement }\end{array}$ & Direction of bead \\
\hline X-ray optics & Parallel beam \\
\hline
\end{tabular}

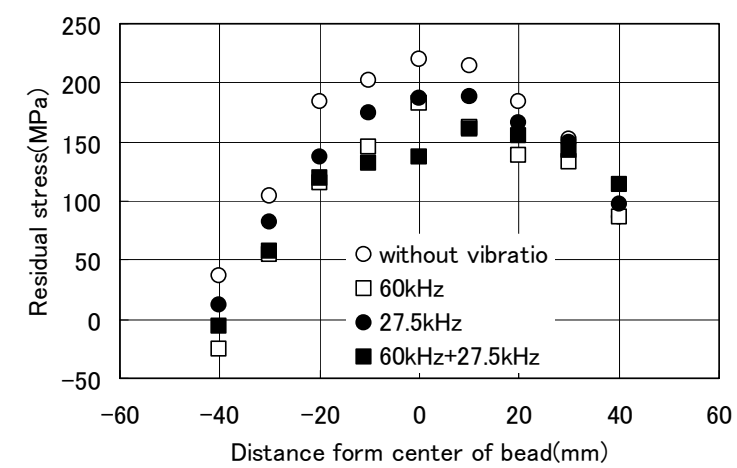

Fig.4 Residual stress on the bead

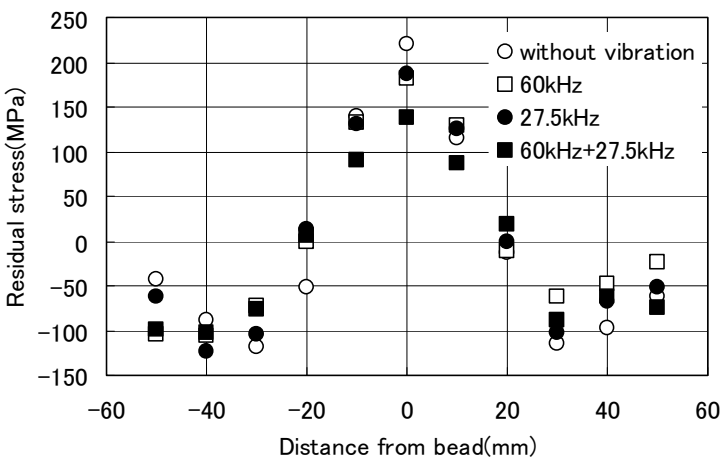

Fig.5 Residual stress in longitudinal direction 


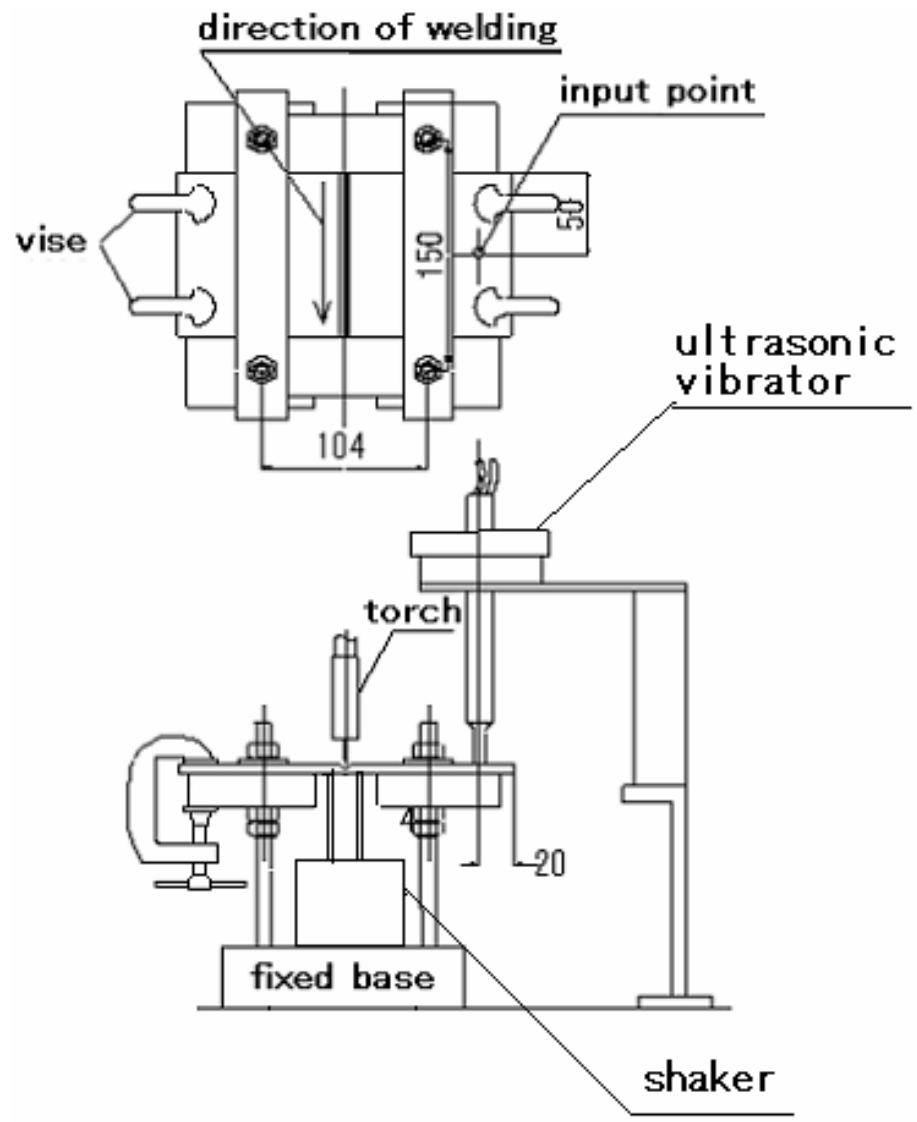

Fig.6 Set of specimens and ultrasonic vibrator and shaker

frequency vibration with natural frequency of the specimen is applied on both plates under the specimens. Reduction of residual stress is examined.

\subsection{Experimental setup}

Size of the specimen is same as $2.1,100 \mathrm{~mm}$ square and $6 \mathrm{~mm}$ thickness. Ultrasonic vibration is used on one plate and low frequency vibration is used on both plates. Low frequency vibration is applied on both plates by the shaker under the plates.

Figure 6 shows experimental setup. The natural frequency of the specimen before welding is $35 \mathrm{~Hz}$. Then, vibration with $35 \mathrm{~Hz}$ is applied below the plate. Frequency of ultrasonic vibration is $60 \mathrm{kHz}$.

\subsection{Results of experiment}

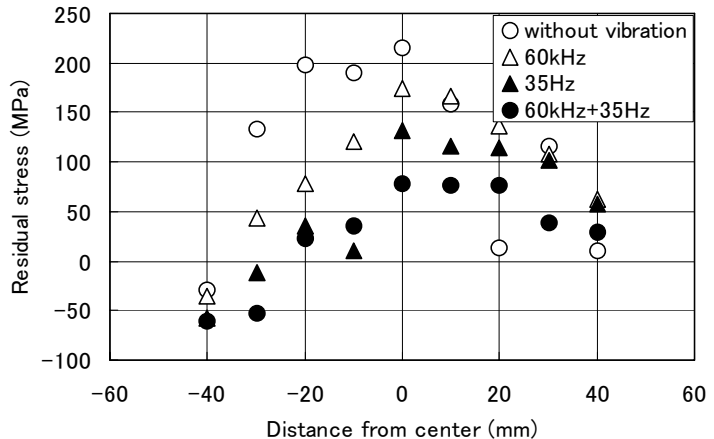

Fig.7 Residual stress on the bead

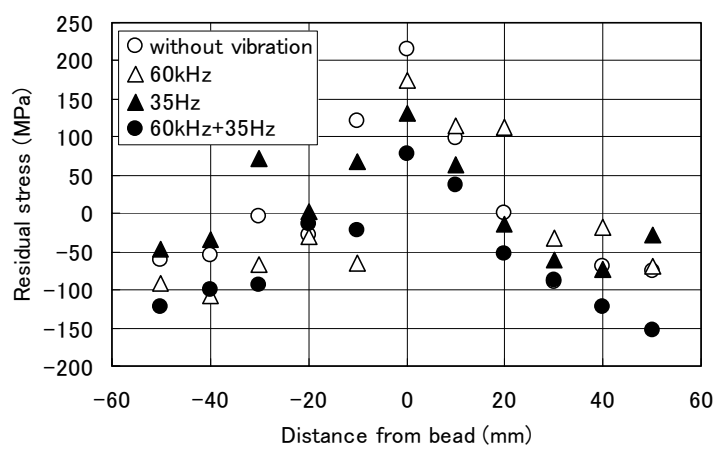

Fig.8 Residual stress in longitudinal direction 
Figure 7 and Figure 8 show residual stresses on the bead and on the line perpendicular to the bead. $\bigcirc$ shows residual stress of specimen welded without vibration. $\triangle$ shows residual stress using $60 \mathrm{kHz}$ ultrasonic vibration on one plate. $\boldsymbol{\Delta}$ shows residual stress using low frequency vibration with $35 \mathrm{~Hz}$ on both plates.

shows residual stress using $60 \mathrm{kHz}$ ultrasonic vibration on one plate and low frequency vibration with $35 \mathrm{~Hz}$ on both plates.

From both figures, when vibration is applied on one plate, tensile residual stress is reduced at center of the bead. Especially, when ultrasonic vibration is applied on one plate and low frequency vibration is applied on both plates, reduction rate of residual stresses is large.

\section{Analytical Method}

Yield stress immediately after welding is very low. Then, plastic deformation is generated by low external load. Residual stress is reduced by release of strain caused by plastic deformation. From simulation method considering this condition, reduction of residual stress near center of the bead is examined qualitatively.

\subsection{Analytical model}

Figure 9 shows two dimensional model. This figure is a model near center of the bead on the specimen. Transverse direction, referred as $\mathrm{x}$-axis, is direction of the line perpendicular to the bead. Vertical direction, referred as $y$-axis, is direction of the bead. In this model, it is considered that vibrations with different frequencies are applied to both edges of the model. However, residual stress at center of the bead is considered. Then, vibrations with different frequencies are applied to an edge of model simultaneously.

As shown in Fig.9(b), vibration is applied in direction of $\mathrm{x}$-axis after springs are extended $Z_{e}$ from equilibrium position in y-axis. $k_{y} Z_{e}$ corresponds to residual stress without ultrasonic vibration. It is assumed that each spring has

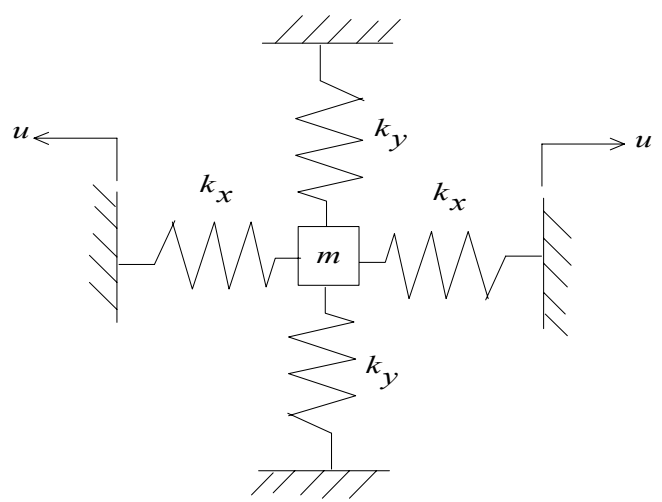

(a) without preload

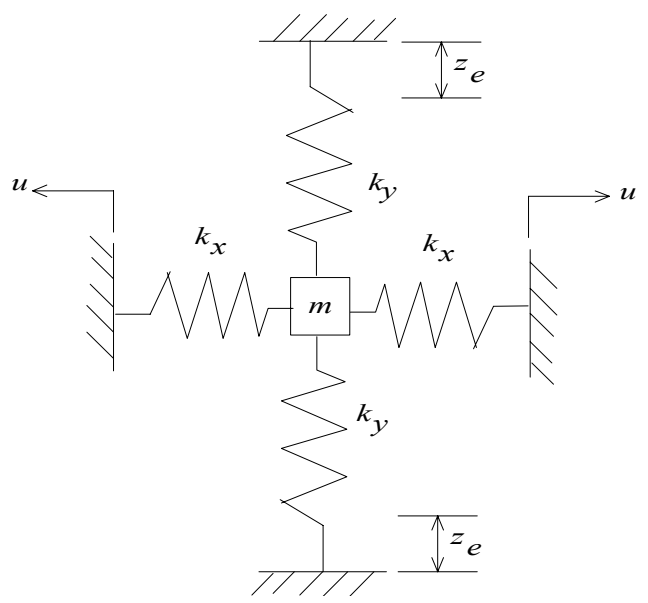

(b) with preload

Fig.9 Two dimensional model

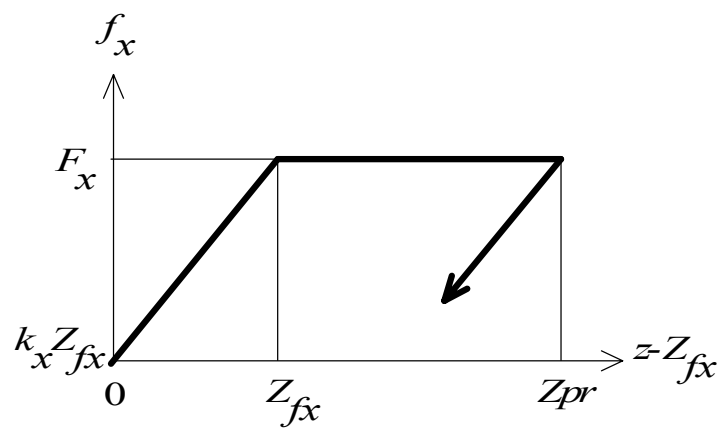

Fig.10 Perfectly-elasto-plastic force-deformation relation 
perfectly-elasto-plastic restoring force-displacement relation. Figure 10 shows relation between restoring force and displacement in $\mathrm{x}$-axis. It is assumed that stresses in direction of $\mathrm{x}$-axis and $\mathrm{y}$-axis are the principle stresses, for example, springs yield under Tresca yield criterion shown in Fig.11.

Let relative displacement of mass to input be $z(x-u)$, equation of motion in $\mathrm{x}$-axis in elastic range is written as:

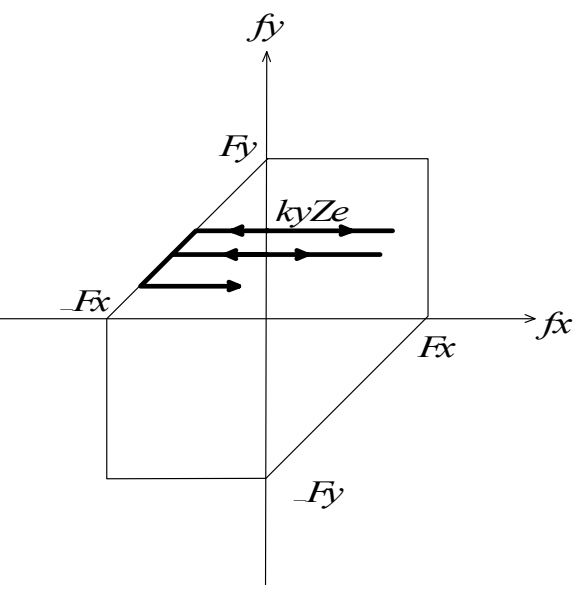

Fig.11 Tresca yield criterion

$$
\ddot{z}+\omega_{n}{ }^{2}\left(z-Z_{p x}\right)=-\ddot{u}
$$

where $Z_{p x}$ is permanent displacement. $\omega_{n}=\sqrt{2 k_{x} / m}$ is the natural circular frequency within elastic limit. Let $Z_{f x}$ is elastic limit displacement, the equation of motion in the case where springs yield when they are subjected to extension force is given as:

$$
\ddot{z}+2 F_{x} / m=-\ddot{u}
$$

where $F_{x}$ is yield force of springs in X-axis. Let $Z_{p r}$ be displacement when $\dot{z}<0, Z_{p x}=Z_{p r}-$ $Z_{f x}$ and the equation of motion within elastic range, Eq.(1), is used.

In the case where springs are subjected to compressive force, $F_{\mathrm{ys}}$ which corresponds to residual stress changes. Then, yield force changes. In this case, the equation of motion is given as:

$$
\ddot{z}-2\left(F_{x}-F_{y s}\right) / m=-\ddot{u}
$$

Let $Z_{e}$ be yield displacement at the beginning of vibration, $F_{y s}$ in the beginning of vibration is $k_{y} Z_{e}$. Let $Z_{m x}$ be displacement when $\dot{z}>0, Z_{p x}=\boldsymbol{Z}_{\boldsymbol{m} \mathbf{r}}+Z_{f x}$ and yield force is given as

$$
f_{x}=\left\{F_{y}-k_{y}\left(Z_{e}+Z_{p y}\right)\right\}
$$

For displacement in y-axis, it is assumed that yield area is equal to width of bead and volume is constant in this area, then

$$
Z_{p y}=-0.5 Z_{p x}
$$

Considering those conditions, the equation of motion within elastic limit Eq.(1) is used. Sum of permanent displacement is $Z_{p y}$. Residual stress after using vibration is obtained as

$$
F_{y f}=k_{y}\left(Z_{e}+Z_{p y}\right)
$$

Stress caused by vibration in $\mathrm{x}$-axis and residual stress in $\mathrm{y}$-axis follows the paths as shown in Fig.11. Whenever permanent displacement occurs, residual stress is reduced. In Fig.11, system yields when system is subjected to compressive stress since probability of yield is very high when system is subjected to compressive stress. In calculation, system always yields when system is subjected to compressive stress.

\subsection{Results of analysis}

Input acceleration $\ddot{u}$ is given as

$$
\ddot{u}=U \sin \omega_{1} t+V \sin \omega_{2} t
$$

where $U$ and $V$ are amplitude of ultrasonic vibration with $60 \mathrm{kHz}$ and $27.5 \mathrm{kHz}$, respectively. $\omega_{1}$ and $\omega_{2}$ are circular frequencies correspond to $60 \mathrm{kHz}$ and $27.5 \mathrm{kHz}$, respectively.

Ratios of residual stress calculated by using ultrasonic vibration to that without vibration are obtained. In Table 2 , results for $60 \mathrm{kHz}$ ultrasonic vibration are shown. In Table 3 , results for $27.5 \mathrm{kHz}$ are shown. $F_{y}$ is yield force for uniaxial stress. The natural frequency of specimen is $35 \mathrm{~Hz}$. From Fig. 4 and Fig.5, reduction rate of residual stress at center of the bead is equal for ultrasonic vibration with $60 \mathrm{kHz}$ and $27.5 \mathrm{kHz}$. Considering this condition 
and a simple relation between $U$ and $V, V=0.5 U$ in Table 3. In this condition, reduction rate is nearly equal for $60 \mathrm{kHz}$ and $27.5 \mathrm{kHz}$. Ratios are less than 1 in all conditions. This means that residual stress is reduced when ultrasonic vibration is used during welding. From Fig.4 and Fig.5, ratios are larger than about 0.7 when one ultrasonic vibration is used. Then, values of $k_{y} Z_{e} / F_{e}$, ratio of initial residual stress to initial yield force, and $F_{y} / m U$, ratio of initial yield force to amplitude of excitation, are selected to obtain ratios larger than about 0.7 .

In Table 4, results are shown when ultrasonic vibrations with $60 \mathrm{kHz}$ and $27.5 \mathrm{kHz}$ simultaneously under the condition $V=0.5 U$. Ratios in Table 4 are less than that in Table 2 and Table 3. From Table 4, it is found that reduction rate is large when ultrasonic vibrations with $60 \mathrm{kHz}$ and $27.5 \mathrm{kHz}$ are used simultaneously.

Ratios of residual stress decrease with decrease of $F_{y} / m U$ or $F_{y} / m V$, that is, increase of amplitude of excitation. This means that residual stress decreases with the increase of amplitude of excitation. This result is the same as vibration with relatively low frequency ${ }^{(6)}$ and random vibration ${ }^{(7)}$. Using vibrations with different frequencies corresponds to increase of amplitude of excitation because the maximum amplitude is sum of amplitudes of vibrations with different frequencies. It is impossible to change amplitude of excitation of ultrasonic vibrator used in this study. However, if it is possible to change amplitude of excitation, it is supposed that residual stress decreases with the increase of amplitude of excitation.

Reduction rate increases with the increase of $k_{y} Z_{e} / F_{y}$, that is, with the increase of residual stress without ultrasonic vibration.

In Table 5, results are shown when ultrasonic vibration with $60 \mathrm{kHz}$ and low frequency vibration with $35 \mathrm{~Hz}$ simultaneously under the condition $V=0.0012 U$. Ratios in Table 5 are less than that in Table 2 when $k_{y} Z_{e} / F_{e}=0.9$ and 0.8 . From Table 5, it is found that reduction rate is large when ultrasonic vibration with $60 \mathrm{kHz}$ and low frequency vibration with $35 \mathrm{~Hz}$ are used simultaneously.

\section{Conclusions}

The effect of vibrations with different frequencies on butt-welding of two thin plates is examined. First, one ultrasonic vibration is applied on one plate and the other ultrasonic vibration with different frequency is applied on the other thin plate. When ultrasonic vibration is not used, high tensile stress is measured at center of the bead. When one 
ultrasonic vibration is used on one plate, residual stress is reduced. However, when ultrasonic vibrations with different frequencies are used simultaneously, reduction rate of residual stress is large. Second, one ultrasonic vibration is applied on one plate and the other vibration with relatively low frequency corresponding to the fundamental natural frequency of the thin plate is applied on both plates. When vibration is applied on one plate, tensile residual stress is reduced at center of the bead. Especially, when ultrasonic vibration is applied on one plate and low frequency vibration is applied on both plates, reduction rate of residual stresses is large. Finally, experimental results are examined by simulation using analytical model considering permanent deformation of springs. It is demonstrated that when ultrasonic vibrations are used during welding, tensile residual stress is reduced.

\section{References}

(1) E.Mele, L.Calado and A.D.Luca,, Experimental Investigation on European Welded Connections, J. Structural Engineering, ASCE, 129-10 (2003), pp.1301-1311.

(2) N.V.Le, Method and Mechanism of Beneficial Residual Stress in Tube, J. Pressure Vessel Technology, ASME, 116-2 (1994), pp.175-178.

(3) K.Nakacho, A Simple Estimating Method for Reduction of Welding Residual Stresses in Thick Welded Joint from Stress-Relief Annealing-Part III: Development of Estimating Equations for Multiaxial Stress State in Thick Welded Joint, J. Pressure Vessel Technology, ASME, 124-1 (2002), pp.14-21.

(4) G.Gnirss, Vibration and Vibratory Stress Relief. Historical Development, Theory and Practical Application, Welding in the World, 26-11/12 (1988), pp.4-8.

(5) S.Aoki, T.Nishimura and T.Hiroi, Reduction of Residual Stress for Welded Joint Using Vibrational Load, Steel and Composite Structure, 4-5 (2004), pp.355-365.

(6) S.Aoki, T.Nishimura, T.Hiroi and S.Hirai, Reduction Method for Residual Stress of Welded Joint Using Harmonic Vibrational Load, Nuclear Engineering and Design, 237 (2007), pp.206-212.

(7) S.Aoki, T.Nishimura and T.Hiroi, Reduction Method for Residual Stress of Welded Joint Using Random Vibration, Nuclear Engineering and Design, 235 (2005), pp.1441-1445.

(8) Electronic Industries Association of Japan, Ultrasonic Engineering, Corona Publishing, Tokyo, (1993), pp.184-188.

(9) S.Aoki, T.Nishimura S.Hirai and T.Hiroi, Effect of Ultrasonic Vibration on Reduction of Residual Stress of Welded Joint, Proc. Asia-Pacific Vibration Conference 2001, 3 (2001), pp.810-814. 